\title{
Evaluation of a group psychoeducational intervention for cancer-related cognitive dysfunction in community-dwelling breast cancer survivors.
}

\author{
Shaikh KT ${ }^{1 *}$, Mamedova $K^{1}$, Rich $\mathbf{J B}^{1}$, Bernstein $\mathbf{L J}^{2,3}$ \\ ${ }^{1}$ Department of Psychology, York University, Toronto, Canada \\ ${ }^{2}$ Department of Supportive Care, Princess Margaret Cancer Centre, Toronto, Canada \\ ${ }^{3}$ Department of Psychiatry, University of Toronto, Toronto, Canada
}

\begin{abstract}
Background: Cancer-related cognitive dysfunction (CRCD) refers to cognitive difficulties experienced by patients following cancer or its treatment. This study reports on a newly developed psychoeducational intervention intended to help breast cancer survivors manage CRCD symptoms.

Objective: We conducted a pilot study to examine preliminary efficacy of this intervention and consider implementation challenges.

Methods: Breast cancer survivors reporting cognitive difficulties were assessed before, immediately after, and one-month following the intervention. Primary outcome measures were participant-reported cognitive function and quality of life. Secondary outcome measures included knowledge of CRCD, memory systems and strategies, and healthy lifestyle activities. Follow-up interviews were conducted.

Results: Five of the seven women who enrolled completed the intervention and three women returned for the one-month post-intervention testing. Immediately post-intervention, participants showed increased confidence in memory abilities, improved functional abilities, and increased CRCD knowledge. Qualitative analyses revealed four themes underscoring participant's experience with this intervention: intentional behavioral changes, normalization and validation, changed relationship to self and others, and perceived lack of support from the medical community.

Conclusion: This pilot study suggests this intervention benefits survivors in multiple ways. A randomized controlled design with a larger sample size is needed to replicate and understand the reliability of changes over time.
\end{abstract}

Keywords: Chemo-brain, Cancer survivorship, Breast cancer, Stress inoculation, Lifestyle, Subjective cognitive complaints, Quality of life.

\section{Introduction}

Many cancer patients experience cognitive impairment during or after cancer treatment. This impairment, referred to as cancer-related cognitive dysfunction (CRCD), may manifest as difficulty with memory, attention, executive functioning, visuo-spatial skills or psychomotor speed [1-3]. Although this phenomenon has been noted in various cancer types [4-6], it is best described in breast cancer patients, where prevalence rates are as high as $78 \%$ [7]. Cognitive dysfunction can persist long after cancer treatment $[7,8]$ at great cost to patients economically, emotionally, and interpersonally [9]. These symptoms can adversely impact participation in everyday life and individuals with CRCD may struggle to fulfill household work [10-13], and relationship obligations [10]. Additionally, individuals with CRCD report a lack of support from the medical community $[10,14]$

Cognitive rehabilitation approaches with demonstrated efficacy in the context of brain injuries [15] have been adapted to target cognitive concerns in cancer survivors $[16,17]$. The Memory and Attention Adaptation Training (MAAT) focuses on selfawareness training, and compensatory strategies [18]. It involves four individual sessions (30-50 min long) every other week with three phone calls in between sessions from a licensed clinical psychologist. A randomized clinical trial found that, compared with waitlisted controls, participants had improved verbal memory performance and patient-reported quality of life [18]. However, patient-reported cognitive abilities did not improve and therefore the intervention may not have been effective at reducing the functional impact of CRCD [18]. In contrast, a group intervention for patients with various cancer sites (e.g. breast, bladder, prostate) that focused on memory techniques found promising improvements in patient-reported cognitive impairment [19]. In a different study, a 6-month course of individual cognitive-behavioral therapy was associated with improvements in self-reported cognitive abilities, but not on objective measures of processing speed [20]. On the other hand, a computerized training program designed to improve executive function (EF), which involved completing 48 sessions with 13 different EF exercises, was associated with improvements in 
objective measures of cognitive flexibility, verbal fluency, and processing speed but not with improvements on a composite score of self-reported assessment of EF [21]. Authors noted that subclinical levels of distress may have impacted subjective assessment of EF and recommended that future programs include relaxation strategies [21].

In a single-arm study, an intervention consisting of a single one-hour individual session for cancer patients selfreporting cognitive dysfunction was shown to increase memory contentment, knowledge of CRCD, and self-efficacy to cope with cognitive symptoms [22]. This intervention provided information on CRCD and compensatory strategies to improve self-management of symptoms. Also, cancer survivors experiencing cognitive complaints may benefit from interventions that target lifestyle components (e.g., exercise, diet, stress), as these factors can impact cognitive performance [23]. Indeed, Henneghan [24] cited lifestyle factors (exercise, stress) as crucial modifiable factors associated with CRCD, however to our knowledge, these factors have not been combined with cognitive rehabilitation techniques to improve management of CRCD.

We developed a group intervention that combines psychoeducation about CRCD with memory strategies, information about lifestyle factors that impact cognition, and stress inoculation skills training. The purpose of the current study was to pilot test it with community-dwelling breast cancer survivors and examine its preliminary efficacy in managing CRCD. Primary quantitative outcomes were participant-reported cognitive function and quality of life. Secondary outcomes were knowledge of CRCD, memory strategies, stress inoculation behaviors, participation in healthy lifestyle activities and overall satisfaction with the intervention. We explored practical concerns, including recruitment, participation, and adherence challenges. Interviews were conducted with participants who completed the intervention to better understand their experiences with this intervention.

\section{Methods}

\section{Participants}

Female breast cancer survivors were passively recruited through the placement of flyers at community sites (e.g., nearby community centers, Gilda's Club Toronto) and local hospital public bulletin boards. Inclusion criteria were completion of cancer treatment $\geq 12$ months prior to the onset of the study (except for hormone therapy) and self-reported cognitive symptoms. Exclusion criteria were a score in the demented range on the Modified [25] Telephone Interview for Cognitive Status (TICS-m) [26], or an inability to communicate in English. No participants had any previous cancer diagnosis or treatment. None of the participants were undergoing hormonal therapy at the time of the intervention.

\section{The intervention}

The intervention consisted of five 2-hour weekly group sessions and a booster session one-month later. Participants received information about CRCD and memory, factors that impact cognition and compensatory strategies. Homework exercises that reviewed each week's session were assigned and required 15-20 minutes to complete. A summary of the intervention's weekly program sessions and booster session is presented in Table 1. The intervention content was adapted from an intervention previously shown to be effective at improving perceived memory ability in older adults [27,28]. The modifications included more cancer-relevant information and inclusion of stress-inoculation training, a CBT-based approach for helping individuals cope with stress. The group leader (KS) had graduate level training in neuropsychology, neuroscience and clinical training in facilitating patient group interventions.

\section{Procedure}

All interested cancer survivors were screened for eligibility via a phone interview during which the TICS-m was administered and medical history and demographic information was collected. Eligible consenting individuals completed assessments at three time-points: (T1) baseline visit before intervention onset; (T2) immediately following the fifth session; and (T3) one-month after T2 (Figure 1). The one-month follow up (T3) testing occurred on the same day as the booster session. Participants who missed a session during the 5-week intervention were given the option of meeting with the group leader within the week to discuss content of missed session. Participants completed a semi-structured interview, lasting 30-45 minutes, 4-5 weeks after the final session of the intervention. Interviews were audiorecorded and transcribed.

\section{Measures}

\section{Primary outcomes}

Participant-reported cognitive function was examined using the Multifactorial Memory Questionnaire(MMQ) and the Functional Assessment for Cancer Therapy-Cognition (FACT-Cog3). The MMQ, a validated measure previously used to assess memory interventions [28,29], assesses satisfaction with memory ability (Contentment, 18 items), attitudes towards memory function (Ability, 20 items) and use of memory strategies (Strategy, 19 items) [29]. Higher scores on these subtests indicate more of the attribute being measured. The FACT-Cog3, a well-validated and commonly used tool designed for cancer populations [19,3032], consists of four subscales: Perceived Cognitive Impairment (20 items), Perceived Cognitive Abilities (9 items), Comments from Others (4 items), and Impact of Perceived Cognitive Impairments on Quality of Life (4 items) [32]. The Perceived Cognitive Impairment subscale was used as the primary score as suggested by test developers [32]. Higher scores on all subscales indicate better cognitive function. Quality of life (QoL) was assessed using the Adapted [33,34]. Illness Intrusiveness Ratings Scale (IIRS, 13 items) [35] which assesses the extent to which symptoms disrupt participation in meaningful activities, with higher scores indicating greater disruption. It consists of five subscales: Physical Well-being and Diet (2 items), Work and Finances (2 items), Marital, Sexual, and Family Relations (3 items), Recreation and Social Relations (3 items), and Other Aspects of Life (3 items).

\section{Secondary outcomes}

Knowledge of CRCD, memory strategies and stress inoculation 
Table 1. Content of intervention weekly sessions and 1-month session.

\begin{tabular}{|c|c|}
\hline Weeks & Content \\
\hline \multirow{3}{*}{1} & Introduction to the group \\
\hline & CRCD and Memory \\
\hline & Homework: Track own memory mistakes \\
\hline \multirow{3}{*}{2} & Factors that affect memory (e.g. medical conditions, medications, diet, exercise, cognitive engagement, attitude, stress) \\
\hline & Relaxation training: deep breathing and visualization \\
\hline & Homework: Track own cognitive and physical activities and relaxation practice \\
\hline \multirow{3}{*}{3} & Overview of memory strategies (e.g. implementation intentions, habits, forming associations, external memory aids, spaced retrieval). \\
\hline & Teaching and practice with associations and spaced retrieval strategy \\
\hline & Homework: Memory strategy practice \\
\hline \multirow{3}{*}{4} & Stress Inoculation Training \\
\hline & Teaching and practice with stress inoculation strategies \\
\hline & Homework: Stress Inoculation strategies practice \\
\hline \multirow{2}{*}{5} & Application of memory strategies in everyday scenarios \\
\hline & Review of previously discussed content \\
\hline \multirow{2}{*}{ Booster } & Overview of CRCD and Memory \\
\hline & Review of memory strategies, stress inoculation techniques and lifestyle behaviours that impact CRCD \\
\hline
\end{tabular}

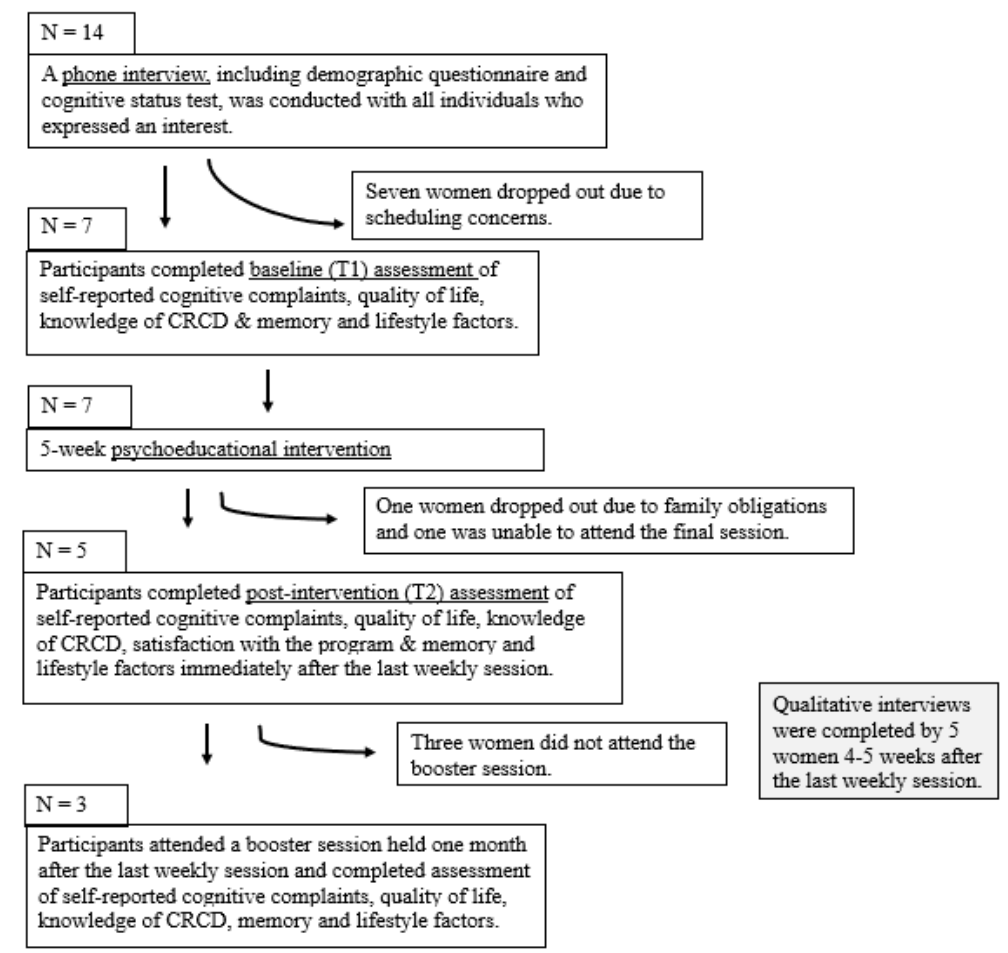

Figure 1. Bilaterally photographed the coronal sections containing the VTA area under 10x magnification at 400 ms exposure.

techniques and participation in brain-healthy lifestyle activities were assessed. Also, we measured overall satisfaction with the intervention. Questionnaires created for this study are provided in Supplementary Materials. The CRCD Knowledge Test, a 12-item short-answer questionnaire, was created to assess knowledge of topics presented in the intervention. Possible scores range from 0 to 12 , with higher scores representing greater knowledge of material. The Memory Strategy Toolbox was a tool used in the Memory and Aging Program [27] as a practical way to measure memory strategy knowledge in everyday life. Participants are presented with memory scenarios and asked to list strategies that would be useful in each scenario, with a maximum total of 12 points overall and higher scores indicating greater knowledge of memory strategies. The
Stressful Situations Scenarios was created for this study to measure strategy knowledge in stressful situations. Participants are presented with stressful scenarios and asked to list strategies which can be useful in those situations. Possible scores range from 0 to 12 and high scores indicate greater knowledge of strategies. A Lifestyle Factors Questionnaire was created for this study and used to measure the number of brain-healthy lifestyle activities that participants engage in. Immediately following the last weekly session of the intervention, participants rated their satisfaction with the 5-week program overall on a single 5-point Likert scale item, anchored by Not Satisfied and Extremely Satisfied. Logistical challenges of delivering the intervention were captured through documentation of recruitment efforts, reasons for decline/withdrawal, and adherence through participation in homework activities and attendance records. 
Citation: Shaikh KT, Mamedova K, Rich JB, et al. Evaluation of a group psychoeducational intervention for cancer-related cognitive dysfunction in community-dwelling breast cancer survivors. J Neurol Neurorehabil Res. 2018;3(1):26-33.

\section{Analyses}

\section{Quantitative analyses}

A paired samples t-test was used to compare scores on outcome measures before the onset of (T1) and immediately after the fifth session (T2). The one-month post-intervention testing scores were not included in statistical analyses because only three people completed that assessment. Effect sizes were calculated using Hedge's gav to account for positive bias in standard mean difference statistics [36,37].

\section{Qualitative analyses}

Interviews were analyzed using QSR International's NVivo 10. An inductive qualitative content analysis was employed to understand the participants' experiences with this intervention. This approach requires a systematic coding and categorization of data to determine patterns such that emerging themes are strongly linked to the data themselves [38]. In developing categories, the comparative method of analysis [39] was used, whereby each meaning unit was continuously compared with other units. This allowed for a rich understanding of the participants' overall experience and ensured that this understanding stayed close to the actual text. Once categorization reached saturation at this level, more abstract themes that subsumed the description categories were developed. These themes were then examined to determine adequate support from the data and confirm that themes supported the research question under investigation [40].

\section{Results}

Demographic and clinical characteristics of participants are presented in Table 2. Mean raw scores for each measure before and immediately after the last program session for those participants who attended the last weekly session are shown in Table 3. Mean raw scores for all participants are reported in supplementary materials (Table S1).

\section{Primary outcome measures}

Immediately following the intervention, participants reported improved memory ability, MT1 $=28.40$ vs. MT2 $=39.20, \mathrm{t}(4)=-$ $8.19, \mathrm{p}=.001$, gav $=0.64$. No significant changes were observed in their memory contentment or strategy use. Participants had improved scores on the Perceived Cognitive Impairments subscale of the FACT-Cog3, MT1=25.80 vs. MT2 39.20, $\mathrm{t}(4)=5.54, \mathrm{p}=.005, \mathrm{gav}=0.61$. There was a trend toward significant T2 improvement on the Impact on Quality of Life subscale $(\mathrm{p}=.057)$. No significant change in IIRS subscale scores was observed post-intervention.

\section{Secondary outcome measures}

Scores on the CRCD Knowledge Quiz increased significantly $(p=.03)$ after the intervention. No improvement in use of strategies was observed on the Memory Toolbox, or the Lifestyle Factor Questionnaire. Improvement in use of stress reduction techniques approached significance $(p=.051)$. Participants expressed satisfaction with the intervention $(\mathrm{M}=4.33$ on a 5-point Likert scale, $\mathrm{SD}=0.82$ ).

\section{Practical issues with intervention delivery}

Despite community advertising in various public locations, only 14 women expressed interest, seven of whom enrolled. The remaining seven were eligible but declined when informed about intervention structure because they could not commit to attending weekly meetings. Of the seven enrolled participants, five were adherent to training as indicated by a high number of sessions attended $(\mathrm{M}=4, \mathrm{SD}=1.15)$ and high percentage of homework completion $(\mathrm{M}=3, \mathrm{SD}=1.15)$. Attendance and participation details are presented in Table 2. Only three participants attended the one-month post-intervention booster session and completed the one-month (T3) assessment. Four women did not attend the booster session or complete T3 assessment, and the reasons given were related to poor health $(\mathrm{N}=1)$, work conflict $(\mathrm{N}=2)$, and no need for review $(\mathrm{N}=1)$. Five women participated in qualitative interviews.

\section{Qualitative results}

All participants described a positive effect from participation in the intervention. Participants commented favorably that the intervention combined information across elements (e.g., memory and CRCD research, stress and lifestyle influences). Four participants reported making healthier lifestyle choices to help manage CRCD symptoms and reported reduced stress following this intervention. Qualitative analyses revealed four themes that highlight participants' experiences with this intervention.

Table 2. Demographics, clinical characteristics and attendance for all participants enrolled $(N=7)$.

\begin{tabular}{|c|c|c|c|c|c|c|c|c|c|c|}
\hline ID & Age & $\begin{array}{c}\text { Time } \\
\text { Since Last } \\
\text { Treatment } \\
\text { (months) }\end{array}$ & $\begin{array}{c}\text { Education } \\
\text { (years) }^{\mathrm{b}}\end{array}$ & $\begin{array}{c}\text { Cognitive } \\
\text { Status } \\
\text { (m-TICS } \\
\text { score) }\end{array}$ & $\begin{array}{c}\text { Employment } \\
\text { Status }\end{array}$ & $\begin{array}{l}\text { Marital } \\
\text { Status }\end{array}$ & $\begin{array}{c}\text { No. Sessions } \\
\text { completed } \\
(\operatorname{Max}=5)\end{array}$ & $\begin{array}{l}\text { No. of } \\
\text { Homework } \\
\text { Activities } \\
\text { Completed } \\
(\operatorname{Max}=4)\end{array}$ & $\begin{array}{l}\text { Booster } \\
\text { Session } \\
\text { Attended? }\end{array}$ & $\begin{array}{c}\text { Interview } \\
\text { Completed? }\end{array}$ \\
\hline 1 & 42 & 28 & 20 & 37 & Homemaker & Married & 2 & 1 & No & No \\
\hline 2 & 67 & 50 & 20 & 38 & Retired & Single & 3 & 2 & Yes & Yes \\
\hline 3 & 46 & 23 & 16 & 36 & On Leave & Single & 4 & 3 & No & Yes \\
\hline 4 & 65 & 23 & 16 & 36 & On Leave & Married & 4 & 3 & No & No \\
\hline 5 & 54 & 101 & 18 & 33 & Unemployed & Married & 5 & 4 & Yes & Yes \\
\hline 6 & 63 & 68 & 16 & 41 & Retired & Married & 5 & 4 & Yes & Yes \\
\hline 7 & 44 & 41 & 12 & 36 & On Leave & Married & 5 & 4 & No & Yes \\
\hline Mean & 54.4 & 47.7 & 16.9 & 36.7 & \multirow{2}{*}{-} & \multirow{2}{*}{-} & 4 & 3 & \multirow{2}{*}{-} & \multirow[b]{2}{*}{-} \\
\hline (SD) & -10.6 & -28.6 & -2.8 & -2.4 & & & -1.15 & -1.15 & & \\
\hline \multicolumn{11}{|c|}{ aTelephone Interview for Cognitive Status - modified version } \\
\hline
\end{tabular}


Table 3. Outcome measures at baseline (T1) and post-intervention (T2) for those participants who completed the intervention.

\begin{tabular}{|c|c|c|c|c|c|}
\hline \multirow{3}{*}{ Outcome Measures } & T1 & $\mathrm{T} 2$ & \multirow{2}{*}{\multicolumn{3}{|c|}{ T1-T2 }} \\
\hline & $(n=5)$ & $(n=5)$ & & & \\
\hline & \multicolumn{2}{|c|}{ Mean (SD) } & $\mathbf{t}$ & p & $\mathbf{g}_{\mathrm{av}}$ \\
\hline \multicolumn{6}{|c|}{ Subjective Cognitive Function } \\
\hline \multicolumn{6}{|c|}{ Multifactorial Memory Questionnaire } \\
\hline Contentment & $28.00(12.78)$ & $25.00(11.70)$ & 0.52 & 0.62 & -0.22 \\
\hline Ability & $28.40(15.11)$ & $39.20(15.44)$ & -8.19 & 0.001 & 0.64 \\
\hline Strategies & $41.80(6.83)$ & $44.20(6.53)$ & -0.99 & 0.378 & 0.32 \\
\hline \multicolumn{6}{|c|}{ FACT-Cog3 Sub-scales } \\
\hline Perceived Cognitive Impairments & $25.80(19.18)$ & $39.20(20.79)$ & -5.54 & 0.005 & 0.61 \\
\hline Impact on Quality of Life & $7.00(4.89)$ & $9.60(6.18)$ & -2.65 & 0.057 & 0.42 \\
\hline Comments from Others & $10.60(6.31)$ & $12.60(3.36)$ & -1.41 & 0.23 & 0.37 \\
\hline Perceived Cognitive Abilities & $15.40(2.97)$ & $20.20(5.97)$ & -1.61 & 0.182 & 0.97 \\
\hline \multicolumn{6}{|c|}{ Quality of Life } \\
\hline \multicolumn{6}{|c|}{ IIIness Intrusiveness Ratings Subscales } \\
\hline Physical Well Being & $9.20(5.02)$ & $9.80(4.92)$ & -0.8 & 0.468 & -0.11 \\
\hline Work \& Finances & $10.80(3.56)$ & $10.80(4.97)$ & 0 & 1 & 0 \\
\hline Marital, Sexual and Family Relations & $8.80(3.49)$ & $5.80(1.79)$ & 2.12 & 0.101 & 1.02 \\
\hline Recreation and Social Relations & $14.20(5.26)$ & $13.80(6.76)$ & 0.27 & 0.799 & 0.06 \\
\hline Other Aspects of Life & $13.00(6.67)$ & $12.00(5.78)$ & 0.42 & 0.697 & 0.16 \\
\hline \multicolumn{6}{|c|}{ Knowledge Tests } \\
\hline Memory Toolbox & $9.00(2.00)$ & $10.80(1.30)$ & -2.01 & 0.105 & 0.99 \\
\hline Stress Toolbox & $3.00(2.34)$ & $6.20(1.64)$ & -2.76 & 0.051 & 1.45 \\
\hline CRCD Knowledge Quiz & $1.10(1.52)$ & $5.20(2.41)$ & $-3.31^{*}$ & 0.03 & 1.88 \\
\hline \multicolumn{6}{|c|}{ Lifestyle Factors } \\
\hline Lifestyle Factors Questionnaire & $12.20(8.41)$ & $10.40(5.18)$ & 0.864 & 0.437 & -0.24 \\
\hline \multicolumn{6}{|c|}{$t:$ Test Statistics; $p$ : probability value; gav Hedge' $\mathrm{g}$; bolded values indicate $\mathrm{p}$-values $<0.05$} \\
\hline \multicolumn{6}{|c|}{$\begin{array}{l}\text { Note: Higher scores on Multifactorial Memory Questionnaire and FACT-Cog3 subscales indicate better memory or cognitive function. Higher scores on Illness Intrusiveness } \\
\text { Ratings Scale subscales indicate lower quality of life. Higher scores on Knowledge Tests indicate greater knowledge. Higher scores on the Lifestyle Factors Questionnaire } \\
\text { indicate more healthy-lifestyle activities. }\end{array}$} \\
\hline
\end{tabular}

\section{Theme: Intentional changes}

Participants commented on the intentional changes they had made to better manage CRCD symptoms. Participants noted that they made more of an effort to remember instead of getting frustrated when confronted with memory difficulties and that they were motivated to exert this effort because they believed it would result in a positive change. "Because that's what I've learned -- that you do really need to try...But the effort is easier if you know it will work. The group showed me it works" (PT \#04). Participants noted greater motivation to keep up with lifestyle activities because of their impact on cognition: "And you know the stress and the diet and the exercise. I think I make more of an effort with that stuff because I know it affects my memory" (PT \#07). Participants commented that they were implementing new memory strategies and that they enjoyed thinking about which strategy would be most effective in each scenario. "It gives me something to do. So, when I have a difficulty remembering...I think of it as a challenge or a game. Like what strategy am I going to use here?” (PT \#02).

\section{Theme: Normalization and validation}

Another theme that emerged was related to the intervention having helped normalize and validate the participants' experiences with changes in their cognitive functioning. Participants described that they were now able to name the condition they were experiencing. "It was nice to put a name to it" (PT \#06). This new knowledge of the condition helped relieve the self-blame that many participants had experienced previously: "It doesn't feel like it's my fault so much anymore"
(PT \#03). Participants expressed that being part of the group helped them realize that they weren't alone in this experience and that others were going through it as well: "It was the first time... really the first time that I felt I was with people who understood it you know...It wasn't just brushing it off. It was like no this is a real thing. And it's not just me. It's us. We all have it" (PT \#07).

\section{Theme: Changed relationship to self and others}

Participants expressed a change in the way they relate to themselves and to others post-intervention. They described more self-confidence in their ability to manage CRCD symptoms: "It feels like I can do this now. I'm definitely more confident in my abilities now" (PT \#02). Participants expressed that knowing about this condition helped them explain it to friends and family which further made it easier to open to those people. "I have the words to describe my experience... So it doesn't feel as hard to talk about it now. I can talk to them about my experience now" (PT \#03).

\section{Theme: Lack of support from medical community}

Some participants expressed a lack of support from the medical community and this intervention seemed to provide acknowledgement that had been lacking from their medical team. They commented that they had not been told about this condition: "You know lots of times with hospitals they don't even acknowledge it. They just refuse to acknowledge it" (PT \#03). "We just weren't told any of this. We were not told. "(PT $\# 07)$. There was also some resentment at the perceived lack of 
progress being made with respect to this condition: "I just think if they [doctors] really wanted to solve this thing they could. They could figure out what it was" (PT \#03).

\section{Discussion}

Preliminary results suggest efficacy of this intervention in improving self-reported memory ability, cognitive impairment, and knowledge of CRCD. Our findings are consistent with previous research that has shown moderate improvements in self-reported abilities after cognitive rehabilitation interventions $[19,20]$. In addition, the mean improvement on the Perceived Cognitive Impairments subscale was 13.4, well above the minimally clinically important difference (MCID; difference of 6.9) [41]. In fact, all participants improved by more than 6.9. Participation in the intervention was not associated with improved scores on QoL measures. Ferguson et al. [18] reported increased quality of life following their intervention, however, only on the Spiritual Well-Being subscale, a dimension not adequately measured in our scales as only one item on the IIRS queried about spiritual activities. Like our results, they did not observe an increase in other aspects of quality of life, including physical and social well-being [18]. Taken together, our findings provide further support that cognitive rehabilitation approaches can improve self-reported cognitive abilities in cancer survivors, but that benefit is not captured by general QoL measures. In addition to cognitive rehabilitation psychoeducation elements, our intervention included stress-inoculation training, as well as education about lifestyle factors that can affect cognition. Scores on the Stressful Situations Scenarios were not significantly different post-intervention. However, the effect size on this change was large and did approach significance $(p=.051)$. It is likely that statistical significance was not achieved because of the small sample size. An alternative explanation may be that a longer course of stress-inoculation training is necessary to effectively integrate skills into daily life. However, most participants reported reduced stress during the interview; thus, they did perceive some stress reduction benefit, whether from the stress inoculation training alone or in combination with other aspects of the intervention. No difference was observed in the Lifestyle Factors Questionnaire. However, during the qualitative interview, most participants reported making healthy lifestyle changes. This discrepancy may be because participants were unaware that their activities were being counted individually and at the T2 assessment, they tended to chunk items together (e.g., writing cardiovascular activities instead of specifying all activities).

Interviews revealed four themes when participants were asked to discuss their experiences of this CRCD intervention. These themes were intentional changes made to manage cognitive dysfunction, normalization and validation of difficulties, changed relationship to self and others and lack of support from the medical community. The first theme indicates the intervention motivated participants to incorporate behavioral changes to improve their cognition. The second theme reveals that the intervention made participants feel "normal" and heard. This is an important benefit considering the perceived lack of support from the medical community described in this study and in others $[10,14]$. This therapeutic benefit has been described in other interventions and suggests that behavioral interventions can promote psychological well-being [42]. Participants also reported changed relationship to self and others, which is an important benefit as CRCD can adversely impact social relationships [12]. In addition, improved sense of self has been posited to improve health functioning due to improved likelihood of participating in health promoting activities [43].

We faced significant recruitment challenges, which may indicate that women struggled to find time to address CRCD symptoms. Half of interested individuals noted that they could not commit to a 5-week intervention due to work or family obligations. In addition, low attendance for the booster session was observed with many women citing other obligations. Despite this, 5 out of 7 participants completed at least four sessions, which suggests that once women enrolled in the intervention, the perceived benefits of attending outweighed the challenges.

There are limitations to the current study that restrict our ability to comment on its efficacy. First, due to recruitment challenges, our sample size was very small, and no control was included in the study design. Thus, post-treatment improvements may reflect intervention expectancies or true intervention-specific effects. Previous similar studies have recruited within cancer center clinics $[18,20]$ which is likely to be more effective, but that approach does not necessarily capture the experiences of cancer survivors who are no longer receiving treatment or being actively followed by their oncology team. Second, participants in this study were enrolled based on self-reported cognitive symptoms rather than objectively measured impairment. This decision was made because objective and subjective measures do not always correlate in this population, and the intervention was designed to improve patient-reported symptoms. Furthermore, reliance on objective measures may cause researchers to miss nuanced cognitive changes that nevertheless impact day-to-day functioning and these objective measures may not be sensitive to cognitive difficulties experienced by cancer survivors $[44,45]$. In addition, such over-reliance on objective measures may have contributed to the historical lack of acknowledgment of CRCD, which continues to anger and upset cancer survivors. Finally, this intervention was designed for breast cancer survivors and therefore future research is needed to determine if such an intervention would be beneficial for people with other cancer sites. The education components specific to breast cancer would require revising, but most of the material, including memory techniques, impact of lifestyle factors on CRCD and stressinoculation training would be relevant regardless of cancer site.

\section{Conclusion}

To our knowledge, this is the first intervention to combine cognitive rehabilitation and relaxation techniques, homework, and stress inoculation training for CRCD. Although our results suggest preliminary efficacy of this intervention in improving management of CRCD symptoms, additional steps, such as offering online formats, must be undertaken to increase the feasibility of this intervention.

\section{Declaration of Interest}

The authors declare that they have no conflict of interest. 


\section{References}

1. Ahles TA, Root JC, Ryan EL. Cancer-and cancer treatmentassociated cognitive change: An update on the state of the science. J Clin Oncol. 2012;30:3675-86.

2. Bernstein LJ, McCreath GA, Komeylian Z, et al. Cognitive impairment in breast cancer survivors treated with chemotherapy depends on control group type and cognitive domains assessed: A multilevel meta-analysis. Neurosci Biobehav Rev. 2017:29:417-28.

3. Vardy J, Rourke S, Tannock IF. Evaluation of cognitive function associated with chemotherapy: A review of published studies and recommendations for future research. J Clin Oncol. 2007;25:2455-63.

4. Meyers CA, Albitar M, Estey E. Cognitive impairment, fatigue, and cytokine levels in patients with acute myelogenous leukemia or myelodysplastic syndrome. Cancer. 2005;104:788-93.

5. Vardy JL, Dhillon HM, Pond GR, et al. Cognitive function in patients with colorectal cancer who do and do not receive chemotherapy: A prospective, longitudinal, controlled study. J Clin Oncol. 2015;33:4085.

6. Zer A, Pond GR, Razak AR, et al. Association of neurocognitive deficits with radiotherapy or chemoradiotherapy for patients with head and neck cancer. JAMA Otolaryngol Head Neck Surg. 2018;144:71-9.

7. Wefel JS, Schagen SB. Chemotherapy-related cognitive dysfunction. Curr Neurol Neurosci Rep. 2012;12:267-75.

8. Koppelmans $\mathrm{V}$, Breteler $\mathrm{M}$, Boogerd $\mathrm{W}$, et al. Neuropsychological performance in survivors of breast cancer more than 20 years after adjuvant chemotherapy. J Clin Oncol. 2012;30:1080-6.

9. McDougall GJ, Oliver JS, Scogin F. Memory and cancer: A review of the literature. Arch Psychiat Nurs. 2014;28:180-6.

10. Boykoff N, Moieni M, Subramanian SK. Confronting chemobrain: An in-depth look at survivors' reports of impact on work, social networks, and health care response. J Cancer Surviv. 2009;3:223-32.

11. Player L, Mackenzie L, Willis K, et al. Women's experiences of cognitive changes or 'chemobrain' following treatment for breast cancer: A role for occupational therapy?. Aust Occup Ther.2014;61:230-40.

12. Von Ah D, Habermann B, Carpenter JS, et al. Impact of perceived cognitive impairment in breast cancer survivors. Eur J Oncol Nurs. 2013;17:236-41.

13. Munir F, Burrows J, Yarker J, et al. Women's perceptions of chemotherapy-induced cognitive side effects on work ability: A focus group study. J Clin Nurs. 2010;19:1362-70.

14. Myers JS. Chemotherapy-related cognitive impairment: The breast cancer experience. Oncol Nurs Forum. 2012:39:1-69.

15. Tsaousides $\mathrm{T}$, Gordon WA. Cognitive rehabilitation following traumatic brain injury: Assessment to treatment. Mt Sinai J Med. 2009;76:173-81.
16. King S, Green HJ. Psychological intervention for improving cognitive function in cancer survivors: A literature review and randomized controlled trial. Front Oncol. 2015;5:72.

17. Zeng Y, Cheng AS, Chan CC. Meta-analysis of the effects of neuropsychological interventions on cognitive function in non-central nervous system cancer survivors. Integr Cancer Ther. 2016;15:424-34.

18. Ferguson RJ, McDonald BC, Rocque MA, et al. Development of CBT for chemotherapy-related cognitive change: Results of a waitlist control trial. Psycho-Oncology. 2012;21:176-86.

19. Cherrier MM, Anderson K, David D, et al. A randomized trial of cognitive rehabilitation in cancer survivors. Life sciences. 2013;93:617-22.

20. Goedendorp MM, Knoop H, Gielissen MF, et al. The effects of cognitive behavioral therapy for postcancer fatigue on perceived cognitive disabilities and neuropsychological test performance. J Pain Symptom Manag. 2014;47:35-44.

21. Kesler S, Hosseini SH, Heckler C, et al. Cognitive training for improving executive function in chemotherapy-treated breast cancer survivors. Clin Breast Cancer. 2013;13:299-306.

22. Bernstein LJ, Dissanayake D, Tirona KM, et al. Investigating the effectiveness of a psycho educational behavioral intervention for cancer-related cognitive dysfunction in women with breast and gynecological cancer: Knowledge, self-efficacy, and behavioral change. Cancer Res._2012:ㄹ.

23. Andreotti C, Root JC, Ahles TA, et al. Cancer, coping, and cognition: A model for the role of stress reactivity in cancerrelated cognitive decline. Psycho-Oncol. 2015;24:617-23.

24. Henneghan A. Modifiable factors and cognitive dysfunction in breast cancer survivors: a mixed-method systematic review. Support Care Cancer. 2016;24:481-97.

25. Brandt J, Spencer M, Folstein M. The telephone interview for cognitive status. Neuropsychiatry Neuropsychol Behav Neurol. 1988;1:111-7.

26. Welsh KA, Breitner JC, Magruder-Habib KM. Detection of dementia in the elderly using telephone screening of cognitive status. Neuropsychiatry Neuropsychol Behav Neurol. 1993:6:103-110

27. Troyer AK. Improving memory knowledge, satisfaction, and functioning via an education and intervention program for older adults. Neuropsychol Dev Cogn B Aging Neuropsychol Cogn. 2001;8:256-68.

28. Wiegand MA, Troyer AK, Gojmerac C, et al. Facilitating change in health-related behaviors and intentions: A randomized controlled trial of a multidimensional memory program for older adults. Aging Mental Hlth. 2013;17:806-15.

29. Troyer AK, Rich JB. Psychometric properties of a new metamemory questionnaire for older adults. J Gerontol: Psychol Social Sciences. 2002;57:19-27.

30. Jacobs SR, Jacobsen PB, Booth-Jones M, et al. Evaluation of the functional assessment of cancer therapy cognitive 
Citation: Shaikh KT, Mamedova K, Rich JB, et al. Evaluation of a group psychoeducational intervention for cancer-related cognitive dysfunction in community-dwelling breast cancer survivors. J Neurol Neurorehabil Res. 2018;3(1):26-33.

scale with hematopoietic stem cell transplant patients. J Pain Symptom Manage. 2007;33:13-23.

31. Vardy J, Wong K, Yi QL, et al. Assessing cognitive function in cancer patients. Support Care Cancer. 2006;14:111-18.

32. Wagner LI, Sweet J, Butt Z, et al. Measuring patient selfreported cognitive function: Development of the functional assessment of cancer therapy-cognitive function instrument. J Support Oncol. 2009;7:32-9.

33. Lorig KR, Sobel DS, Ritter PL, et al. Effect of a selfmanagement program on patients with chronic disease. Eff Clin Pract. 2001;4:256-62.

34. Stanford Patient Education Research Center (2010) Adapted illness intrusiveness ratings. Retrieved November 20, 2017,

35. Devins GM, Mandin H, Hons RB, et al. Illness intrusiveness and quality of life in end-stage renal disease: Comparison and stability across treatment modalities. Health Psychol. 1990;9:117-42.

36. Durlak JA. How to select, calculate, and interpret effect sizes. J Pediatr Psychol. 2009;34:917-28.

37. Lakens D. Calculating and reporting effect sizes to facilitate cumulative science: A practical primer for t-tests and ANOVAs. Front Psychol. 2013;4:863-76.

38. Vaismoradi M, Jones J, Turunen H, et al. Theme development in qualitative content analysis and thematic analysis. J Nurs Edu. 2016;6:100-7.

39. Fram SM. The constant comparative analysis method outside of grounded theory. Qual Report. 2013;18:1-25.

40. Braun V, Clarke V. Using thematic analysis in psychology. Qual Res Psychol. 2006;3:77-101.

41. Cheung YT, Foo YL, Shwe M, et al. Minimal clinically important difference (MCID) for the functional assessment of cancer therapy: Cognitive function (FACT-Cog) in breast cancer patients. J Clin Epidemiol. 2014;67:811-20.

42. Vandermorris S, Davidson S, Au A, et al. Accepting where I'm at a qualitative study of the mechanisms, benefits, and impact of a behavioral memory intervention for communitydwelling older adults. Aging Ment Health. 2017;21:895-901.

43. Bandura A. Health promotion by social cognitive means. Heal Edu Behav. 2004;31:143-64.

44. Pullens MJ, De Vries J, Roukema JA. Subjective cognitive dysfunction in breast cancer patients: A systematic review. Psycho Oncol. 2010;19:1127-38.

45. Yao C, Rich JB, Tirona $\mathrm{K}$, et al. Intra-individual variability in reaction time before and after neoadjuvant chemotherapy in women diagnosed with breast cancer. Psych Oncol. 2017;26:2261-8

\section{*Correspondence to:}

Komal T. Shaikh

Department of Psychology

York University

Toronto

Canada

Tel: 416-577-0723

E-mail: kshaikh@yorku.ca 